DRAFT VERSION FEBRUARY 5, 2008

Preprint typeset using $\mathrm{LT}_{\mathrm{E}} \mathrm{X}$ style emulateapj v. 6/22/04

\title{
FOSSILS OF REIONIZATION IN THE LOCAL GROUP
}

\author{
Nickolay Y. GNEdin ${ }^{1,3}$ And Andrey V. KRAVTSOV ${ }^{2,3}$ \\ Draft version February 5, 2008
}

\begin{abstract}
We use a combination of high-resolution gasdynamics simulations of high-redshift dwarf galaxies and dissipationless simulations of a Milky Way sized halo to estimate the expected abundance and spatial distribution of the dwarf satellite galaxies that formed most of their stars around $z \sim 8$ and evolved only little since then. Such galaxies can be considered as fossils of the reionization era, and studying their properties could provide a direct window into the early, pre-reionization stages of galaxy formation. We show that $\sim 5-15 \%$ of the objects existing at $z \sim 8$ do indeed survive until the present in the Milky Way like environment without significant evolution. This implies that it is plausible that the fossil dwarf galaxies do exist in the Local Group. Because such galaxies form their stellar systems early during the period of active merging and accretion, they should have spheroidal morphology regardless of their current distance from the host galaxy. Their observed counterparts should therefore be identified among the dwarf spheroidal galaxies. We show that both the expected luminosity function and spatial distribution of dark matter halos which are likely to host fossil galaxies agree reasonably well with the observed distributions of the luminous $\left(L_{V} \gtrsim 10^{6} L_{\odot}\right)$ Local Group fossil candidates near the host galaxy $(d \lesssim 200 \mathrm{kpc})$. However, the predicted abundance is substantially larger (by a factor of 2-3) for fainter galaxies $\left(L_{V}<10^{6} L_{\odot}\right)$ at larger distances $(d \gtrsim 300 \mathrm{kpc})$. We discuss several possible explanations for this discrepancy.
\end{abstract}

Subject headings: cosmology: theory - galaxies: dwarf - galaxies: evolution - galaxies: formation - stars: formation - galaxies: halos - methods: numerical

\section{INTRODUCTION}

Abundance and spatial distribution of the faintest dwarf galaxies provide information and constraints on the galaxy formation processes and may give important clues to the nature of dark matter. Cosmological simulations (Klypin et al. 1999; Moore et al. 1999) and semi-analytic models of galaxy formation (Kauffmann et al. 1993; Bullock et al. 2000; Somerville 2002; Benson et al. 2002) predict the number of gravitationally bound, dark matter clumps (or subhalos) around the Milky Way type galaxies larger, by up to an order of magnitude, than the observed number of dwarf galaxies in the Local Group. In addition, the predicted spatial distribution of the dark matter subhalos around their hosts is more extended than the observed distribution of the Local Group satellites (Tavlor et al. 2004; Kravtsov et al. 2004), indicating that strong spatial bias in galaxy formation should have been present if the CDM scenario is correct.

Formation of galaxies in small-mass, dwarf-sized halos should be affected by heating from the ultra-violet (UV) radiation and internal feedback from stars. UV heating, in particular, is expected to be highly important after the epoch of reionization when the entire volume of the Universe is affected by the cosmic UV background. The UV heating leads to dramatic increase of the characteristic Jeans or filtering mass, $M_{\mathrm{F}}$, of intergalactic gas (Gnedin \& Hui 1998; Gnedin 2000). The dark matter halos with masses $M \lesssim M_{\mathrm{F}}$ (or circular velocities $V_{\mathrm{c}} \lesssim V_{\mathrm{F}}$ ) cannot accrete new gas and therefore do not have supply of fresh gas to fuel the continuing star formation

\footnotetext{
${ }^{1}$ Particle Astrophysics Center, Fermi National Accelerator Laboratory, Batavia, IL 60510, USA; gnedin@ fnal.gov

2 Kavli Institute for Cosmological Physics and Enrico Fermi Institute, The University of Chicago, Chicago, IL 60637 USA; andrey@oddjob.uchicago.edu

${ }^{3}$ Department of Astronomy \& Astrophysics, The University of Chicago, Chicago, IL 60637 USA
}

(e.g., Efstathiou 1992; Thoul \& Weinberg 1996; Ouinn et al. 1996; Navarro \& Steinmetz 1997; Kitavama \& Ikeuchi 2000; Gnedin 2000; Dijkstra et al. 2004). In addition, the UV background can evaporate the existing gas in small halos (Barkana \& Loeb 1999; Shaviv \& Dekel 2003; Shapiro et al. 2004), which would also reduce the star formation rate.

In the presence of these galaxy formation suppression processes, the smallest dwarf galaxies can form: (1) if their host halos have formed sufficiently early, before the reionization epoch and UV heating became significant (Bullock et al. 2000) and/or (2) if the host halos become sufficiently massive after reionization such that UV heating effects are not significant (Kravtsov et al. 2004). It is of course possible that a halo forms early and forms stars before reionization and then stays above filtering mass for an extended period of time after reionization due to continuing merging and accretion. Such galaxies would exhibit continuous, albeit perhaps episodic (see Kravtsov et al. 2004), star formation over an extended period of time.

It is also possible that some galaxies form stars before reionization and then not evolve much in terms of their total and stellar mass, with their stellar populations evolving mostly passively. Most of such galaxies do not survive until $z=0$ but are disrupted via merging and tidal heating, contributing to the formation of the bulge, stellar halo, and halo population of globular clusters of the host galaxies (e.g., White \& Springel 2000; Bullock et al. 2001; Kravtsov \& Gnedin 2005; Bullock \& Johnston 2005; Robertson et al. 2005; Moore et al.|2005). However, a fraction of them can survive. Ricotti \& Gnedin (2005) found that many of the dwarf galaxies identified in the high-resolution gas dynamic simulation at $z \approx 8$ do indeed resemble a subset of the observed old dwarf spheroidal galaxies in the Local Group in their kinematic, structural, and chemical properties.

Such galaxies would then represent fossils of the reionization era, and studying their properties could provide a direct 
window into the early, pre-reionization stages of galaxy formation. As such, it would be extremely interesting to identify and study this population in the Local Group. In this paper, we present theoretical estimates for the abundance and spatial distribution of the fossil galaxies using a combination of sophisticated galaxy formation simulations at high redshifts, which include non-equilibrium chemistry and self-consistent, three-dimensional radiative transfer and high-resolution dissipationless simulations of a Milky Way-sized halo, which allows us to track small-mass dwarf dark matter halos from the pre-reionization epoch $(z \sim 10)$ to their present-day Milky Way-like environment.

\section{METHOD}

\subsection{Simulations}

In this paper we combine results from several simulations to connect models of high-redshift dwarf galaxies with the present-day Local Group. The simulation we use for modeling high-redshift dwarf galaxies is described in detail in Ricotti et al. (2002a b) as run "256L1p3" and was used in Ricotti \& Gnedin (2005) as the main basis for their conclusions.

The simulation includes dark matter, gas, stars, the feedback of star formation on the gas chemistry and cooling, and the spatially-inhomogeneous and time dependent radiative transfer of ionizing and Lyman-Werner band photons - the latter being crucial for realistic modeling early star formation. The run followed $256^{3}$ dark matter particles, an equal number of baryonic cells, and more than 700,000 stellar particles. The mass resolution of the simulation is $900 M_{\odot}$ in baryons, and real comoving spatial resolution (twice the Plummer softening length) is $150 h^{-1} \mathrm{pc}$ (which corresponds to a physical scale of $24 \mathrm{pc}$ at $z=8.3$ ) in a computational box with of $1 h^{-1} \mathrm{Mpc}$ on a side. Hereafter, we refer to this simulation as L1.

Clearly, the $1 h^{-1} \mathrm{Mpc}$ box is too small to be a representative volume of the universe at any moment in time. The box is appropriate for the purposes of this study because properties of the small-mass dwarf galaxies formed in this simulation are determined primarily by the physics of gravitational collapse, gas cooling, star formation and feedback and should not be affected by the box size. However, these galaxies would not be able to reionize even such a small volume of space. Therefore, Ricotti \& Gnedin (2005) introduced an additional source of ionizing radiation within the computational box, corresponding to a star-forming galaxy with constant star formation rate of $1 M_{\odot} /$ yr. The source was switched on at $z=9.0$, and by $z=8.3$ the entire simulation box was completely ionized. At that time, the computational volume contains about two dozen galaxies with luminosities between $10^{5}$ and $10^{7} L_{\odot}$ and circular velocities $\lesssim 30 \mathrm{~km} / \mathrm{s}$. These galaxies lost their diffuse IGM due to the UV feedback of reionization (Gnedin 2000), and stopped forming stars. Ricotti \& Gnedin (2005) argue that these galaxies can be identified with the population of fossil galaxies in the Local Group dwarfs.

The specific value for the redshift at which $1 h^{-1} \mathrm{Mpc}$ box is reionized $(z=9)$ is, of course, somewhat arbitrary, but it is important to note that it should not be identified with the redshift of reionization of the entire universe. It is likely that the region around the Local Group progenitor is reionized considerably earlier than the universe as a whole, because progenitors of the Milky Way-sized objects can easily create their own $\mathrm{H}$ II regions of the order of $1 h^{-1} \mathrm{Mpc}$ by $z \sim 9$ or even earlier ${ }^{4}$.

A $1 h^{-1} \mathrm{Mpc}$ box size is also too small to be representative of the Local Group environment. In the concordance LCDM cosmology ${ }^{5}$ adopted throughout this paper, this box contains only $1.2 \times 10^{11} M_{\odot}$, whereas the mass of the Local Group is estimated to be about $3 \times 10^{12} M_{\odot}$ (e.g., Klypin et al. 2002). In order to make a connection between $z=8$ universe and the Milky Way-like environment at $z=0$, we use a dissipationless (dark matter only) simulation with $20 h^{-1} \mathrm{Mpc}$ box (hereafter L20), which was run using the Adaptive Refinement Tree $N$-body code (ART, Kravtsov et al. 1997; Kravtsov 1999) to follow the evolution of the Milky Way-sized halo with high resolution. A Lagrangian region corresponding to a sphere of $5 R_{\text {vir }}$ of a Milky Way-sized halo at $z=0$ in a low-resolution simulation was resimulated with higher resolution using multiple mass resolution technique (this simulation is Box20 run presented in Prada et al. 2005). In the high-resolution region the mass of the dark matter particles is $m_{\mathrm{p}}=6.1 \times 10^{5} h^{-1} M_{\odot}$, corresponding to effective $1024^{3}$ particles in the box, at the initial redshift of the simulation $\left(z_{\mathrm{i}}=70\right)$. The high mass resolution region was surrounded by layers of particles of increasing mass with a total of four particle species. The simulation starts with a uniform $256^{3}$ grid covering the entire computational box. Higher force resolution is achieved in the regions corresponding to collapsing structures by recursive refining of all such regions using an adaptive refinement algorithm. Only regions containing highest resolution particles were adaptively refined. The maximum of nine refinement levels was reached in the simulation corresponding to the peak formal spatial resolution of $150 \mathrm{~h}^{-1}$ comoving parsec. The Milky Way-sized host halo has the virial mass of $1.4 \times 10^{12} h^{-1} M_{\odot}$ (or 2.3 million particles within the virial radius) and virial radius of $230 h^{-1} \mathrm{kpc}$ at $z=0$.

\subsection{Simulation and Data Samples}

Before we compare results of the cosmological simulation with observational data, we consider the limits of its numerical resolution. In this paper we choose to characterize dark matter halos by their maximum circular velocity $V_{\max }$, because it is well-defined while definition of the halo mass is rather arbitrary. For the low-mass halos that may host dwarf galaxies, the maximum circular velocity function (the number of halos per unit $V_{\max }$ ) is expected to be close to a powerlaw (e.g., Klypin et al. 1999). Thus, strong deviation from a power-law at small halo masses indicates resolution limit of the simulation (see, e.g., Reed et al. 2005).

Figure1illustrates this point. It shows the cumulative maximum velocity function (i.e., the number of dark matter halos with $V_{\max }$ above a given value) at $z=8$ and $z=0$ for the L20 run. The strong deviation from the power-law at $z=8$ can be seen at $V_{\max } \lesssim 13 \mathrm{~km} / \mathrm{s}$, indicating the resolution limit of the L20 simulation at this epoch. At $z=0$, the issue is more complicated because resolution depends on the distance to the Milky Way-sized halos halo. For example, an isolated halo will exist even if its internal structure is poorly resolved, but it may be prematurely disrupted when it falls into a parent halo and experiences tidal heating. Three lines in the lower panel of Fig. 11 do indeed show that while the L20 simulation is sufficient to resolve dark matter halo down to about $8 \mathrm{~km} / \mathrm{s}$

\footnotetext{
${ }^{4}$ This indeed happens in a gasdynamics simulation of a Milky Way-sized progenitor with radiative transfer simulation analyzed by the authors (the simulation is described briefly in Tasitsiomi 2006).

${ }^{5}\left(\Omega_{\mathrm{m}}, \Omega_{\Lambda}, h, \sigma_{8}\right)=(0.3,0.7,0.7,0.9)$.
} 


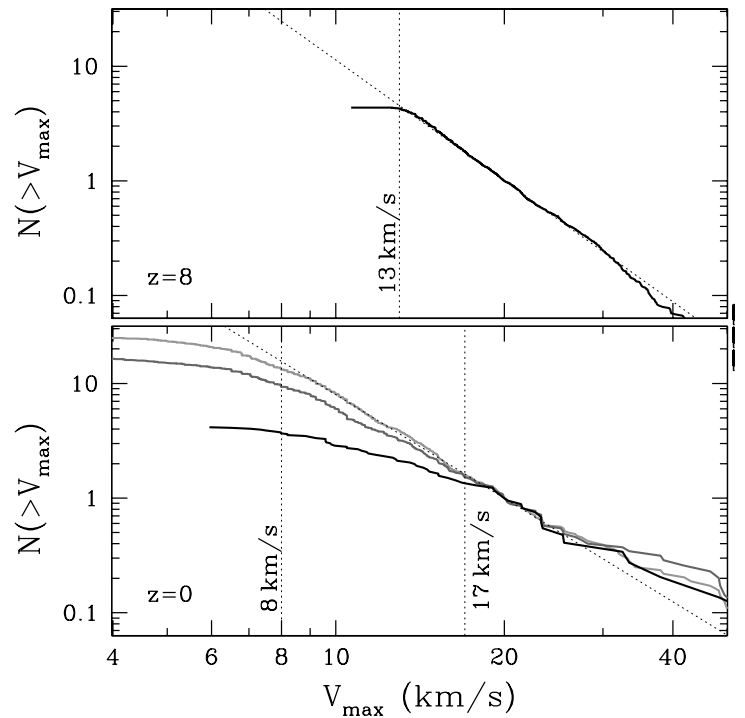

FIG. 1.- Cumulative maximum circular velocity functions for the L20 run at $z=8$ (top panel) and $z=0$ (bottom panel). Velocity functions at $z=0$ are shown for three subsamples: halos within $1 \mathrm{Mpc}$ of the parent halo (light gray line), $300 \mathrm{kpc}$ (dark gray line), and $100 \mathrm{kpc}$ (black line). Tilted thin dotted lines show power-law $N\left(>V_{\max }\right) \propto V_{\max }^{3}$, while the vertical dotted lines mark characteristic values of $V_{\max }$ corresponding to the resolution limit of the simulation, as discussed in the text. The velocity functions in both panels are normalized to unity at $V_{\max }=20 \mathrm{~km} / \mathrm{s}$.

within $1 \mathrm{Mpc}$ of the parent halo ${ }^{6}$, it is complete only down to $17 \mathrm{~km} / \mathrm{s}$ for halos within $100 \mathrm{kpc}$ of the Milky Way-sized halo.

We can simply correct for the incompleteness by a velocity dependent factor that is required to restore the power-law form of $N\left(>V_{\max }\right)$ function (in other words, the correction factor is the ratio of the power-law and the actual velocity function measured in simulations). To illustrate the effect of incompleteness we will use both the uncorrected and the corrected samples, which should bracket the correct result. As we show below, our results are not highly sensitive to this correction even for the $d<100 \mathrm{~km} / \mathrm{s} \mathrm{sample}$, for which the correction is the largest. Note also that the correction only becomes large at $V_{\max } \lesssim 15 \mathrm{~km} / \mathrm{s}$, while none of the observed dwarf galaxies in the Local Group are likely to have $V_{\max }$ significantly below $15 \mathrm{~km} / \mathrm{s}$ (e.g., Stoehr et al. 2002; Zentner \& Bullock 2003; Kazantzidis et al. 2004; (Moore et al. 2005)

Figure 2 shows the sample of dark matter halos from the L20 run we use in this paper. Three shades of gray show three subsamples according to the distance from the parent halo at $z=0$. As we mentioned above, we only select halos that have $V_{\max }>13 \mathrm{~km} / \mathrm{s}$ at $z=8$, so our sample is complete at high redshift. For these halos, $V_{\max }$ at $z=0$ reaches down to $V_{\max } \approx 8 \mathrm{~km} / \mathrm{s}$, so our sample of halos with $d<1 \mathrm{Mpc}$ is effectively complete, while subsamples with smaller limits on $d$ are incomplete to various degrees.

Finally, the L1 simulation allows us to relate the stellar luminosities of model dwarf galaxies to the maximum circular velocities of their dark matter halos at $z=8$. The latter are measured with the same halo finder algorithm used to identify halos in the L20 run. Figure 3 shows the distribution of these two properties for the model dwarf galaxies from the $\mathrm{L} 1$ run. We marked the completeness limit of the L20 run at

${ }^{6}$ The improvement in resoltion limit, from $13 \mathrm{~km} / \mathrm{s}$ at $z=8$ to $8 \mathrm{~km} / \mathrm{s}$ at $z=0$, is likely due to the fact that in a adaptive mesh refinement simulation we use, the resolution becomes somewhat better with time.

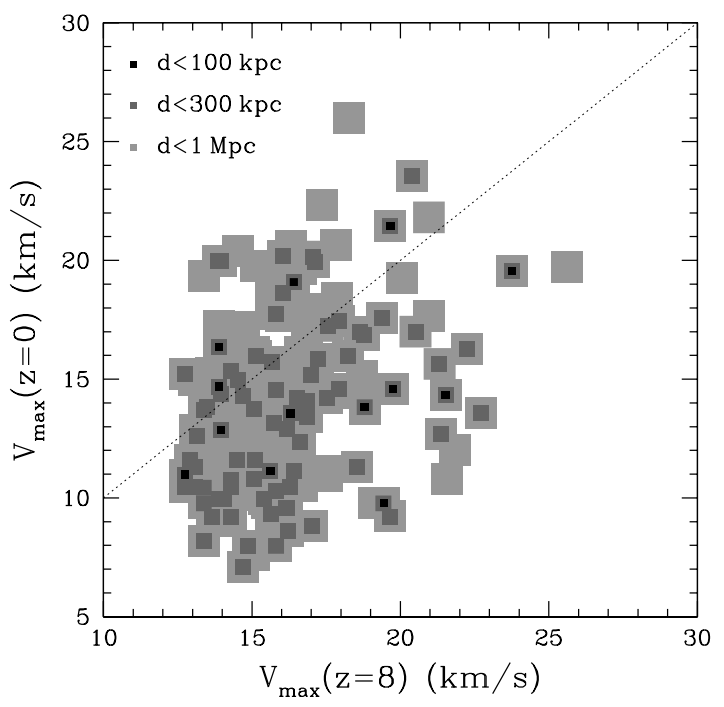

FIG. 2.-Distribution of maximum circular velocities for dark matter halos from run L20 at $z=8$ versus $z=0$. Halos at $z=0$ are distinguished by their distance to the parent halo: halos within $1 \mathrm{Mpc}$ (large light gray squares), within $300 \mathrm{kpc}$ (intermediate darker gray squares), and within $100 \mathrm{kpc}$ (small black symbols). Only halos with $V_{\max }(z=8)>13 \mathrm{~km} / \mathrm{s}$ are shown.

$z=8\left(V_{\max }=13 \mathrm{~km} / \mathrm{s}\right)$ with the thin dotted line, and throughout this paper we only use dwarf galaxies above this limit. Fortunately, all model dwarfs with $L_{V}>10^{5} L_{\odot}$ fall within this range.

The objects shown in Figures 2 and 3 thus, constitute the sample of the simulated galaxies that we use in this paper. Our main task now is to compare these model galaxies to the observed dwarf galaxies in the Local Group that Ricotti \& Gnedin (2005) identified as reionization fossils. The observed sample includes Draco, Phoenix, Sculptor, Sextans, Tucana, and Ursa Minor for the Milky Way subgroup, and And I-III,V,VI,IX, Antlia, Cassiopea, Cetus, EGB0427, and SagDIG for the Andromeda subgroup. These galaxies are selected because they are dim (V-band luminosity below $\left.10^{7} L_{\odot}\right)$ and because they formed at least $70 \%$ of their stars in one single burst at an early epoch (which is identified with the reionization epoch). These galaxies therefore represent reasonable candidates for the reionization fossils. This identification is, of course, somewhat uncertain because the absolute ages of $10-12 \mathrm{Gyr}$ old stars are not measured with sufficient accuracy. Nevertheless, we will assume in the remainder of this paper that the observational samples are not highly contaminated by non-fossil galaxies. We refer reader to Ricotti \& Gnedin (2005) for more details on how the observational sample is selected.

\subsection{Survival Probability}

In order to test the fossil scenario, we need first to measure the probability for a low-mass dark matter halo to remain relatively intact (i.e., not to evolve significantly) from $z \sim 8$ until the present time, which Ricotti \& Gnedin (2005) called the "survival probability." We use the L20 simulation to measure the survival probabilities for dark matter halos. Since in the dissipationless simulation we cannot identify halos with luminous galaxies, we can only measure the survival probability as a function of the maximum circular velocity. We will use the mapping between circular velocities and galaxy luminosities provided by the L1 run (see Figure 3) to calculate from $V_{\max }$ 


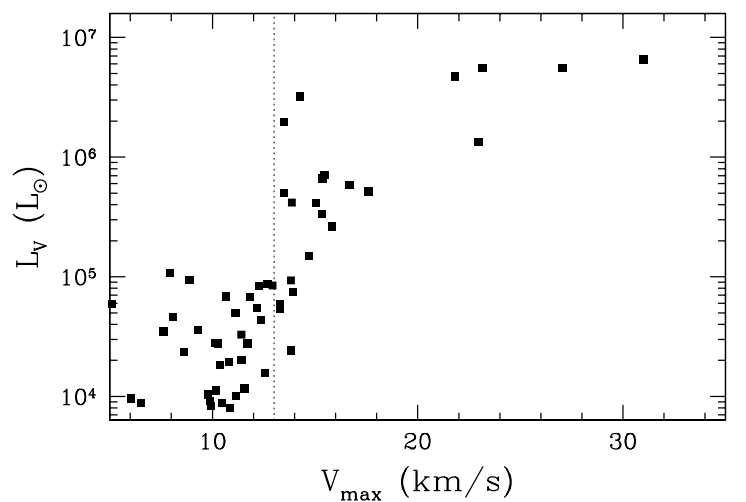

FIG. 3.- The luminosity - maximum circular velocity relation for the dwarf galaxies in the run $\mathrm{L} 1$ at $z=8$. The thin dotted line marks the resolution limit of our sample at $V_{\max }=13 \mathrm{~km} / \mathrm{s}$ (see text for details).

to luminosity.

We measure the survival probability by tracking evolution of the individual halos from $z=8$ to the present time ${ }^{7}$ using the method developed by Kravtsov et al. (2004). To track the halos we use 156 simulation outputs equally spaced in expansion factor from $z=14$ to $z=0$.

The tracks then can be used to estimate the fraction of halos existing at $z=8$ which remain distinct, bound objects at $z=0$. For detailed comparison with observations, it is useful also to differentiate halos with respect to the distance to the Milky Way-sized progenitor, as significant morphological segregation is known to exist in the Local Group. Thus, we define the survival probability of halos within a given distance $d$ (at $z=0)$ from the parent halo as

$$
P_{\mathrm{S}}\left(d, V_{\max }\right)=\frac{N_{\mathrm{H}}\left(8 \rightarrow 0, V_{\max }\right)}{N_{\mathrm{H}}\left(z=8, V_{\max }\right)},
$$

where $N_{\mathrm{H}}\left(8 \rightarrow 0, V_{\max }\right)$ is the number of halos that exist both at $z=0$ and $z=8$ within distance $d$ (measured at $z=0$ ) from the parent halo as a function of $V_{\max }$, and $N_{\mathrm{H}}\left(z=8, V_{\max }\right)$ is the total number of halos at $z=8$ located in the region of space (the "Lagrangian region") that ends up as a sphere of radius $d$ centered on a parent halo at $z=0$.

It is important to stress that in equation (10 the distance $d$ is measured at $z=0$, while the maximum circular velocity $V_{\max }$ is measured at $z=8$. Operationally, in order to find all halos within the $z=8$ Lagrangian region of sphere of radius $d$ around the Milky Way-sized halo at $z=0$, we tag all dark matter particles within the sphere and identify all dark matter halos at $z=8$ located in the region of space occupied by the tagged dark matter particles.

Note that in this study we calculate and apply the survival probability to the small-mass dwarf objects of $V_{\max } \lesssim$ $30 \mathrm{~km} / \mathrm{s}$. Such galaxies are dark matter dominated and effects of baryon dissipation on the inner mass distribution, $V_{\max }$, and on the survival of halos at small distances to the host (e.g., Macciò et al. 2005) should be small.

As an example, Figure 4 shows the survival probability $P_{\mathrm{S}}$ as a function of $V_{\max }(z=8)$ for all dark matter halos from the L20 run. The survival probability ranges from a few to $\approx 25$ per cent and there is a clear trend with the distance to the parent halo. Our measurement of $P_{\mathrm{S}}\left(V_{\max }\right)$ becomes highly noisy for $V_{\max }>35 \mathrm{~km} / \mathrm{s}$ due to small numbers of halos.

\footnotetext{
${ }^{7}$ In the next section we discuss the sensitivity of our results to the specific choice of the redshift at which fossils are identified.
}

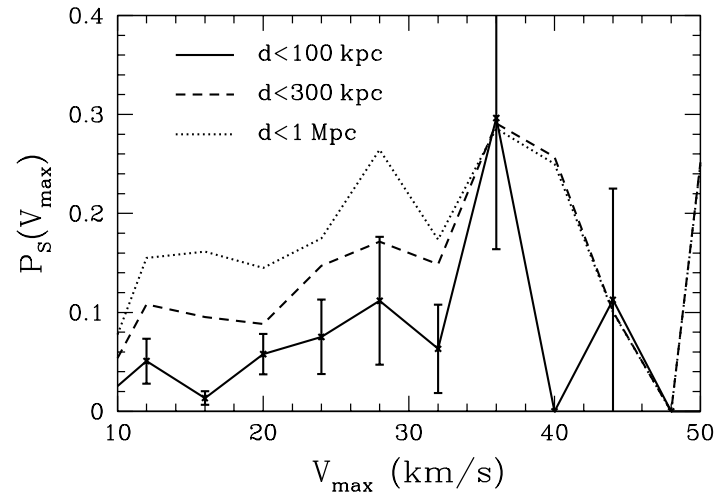

FIG. 4.- The survival probability, $P_{\mathrm{S}}\left(V_{\mathrm{max}}\right)$, of all dark matter halos that end up within $100 \mathrm{kpc}$ (solid line), $300 \mathrm{kpc}$ (dashed line), and $1 \mathrm{Mpc}$ (dotted line) from the parent halo in the L20 run. The error bars are Poisson only. (Reminder: the maximum circular velocity $V_{\max }$ is measured at $z=8$.)

\subsection{Defining a Fossil}

The survival probability is of course defined for all dark matter halos, not only for those that host fossil galaxies. In order to compute the survival probability for fossils only, we need an operational definition of a reionization fossil applicable to dissipationless simulations. We consider a dwarf galaxy to be a fossil if it experiences little or no star formation after reionization. This would be the case if the galaxy does not accrete fresh gas to fuel its continuing growth and star formation. The accretion of gas on dark matter halos is controlled by the filtering mass (Gnedin 2000), which is easily calculable for a representative volume of the universe, but is not well defined for a region that collapses into a Milky Way-sized galaxy. As we noted above, such region can be reionized considerably earlier. Nevertheless, after the region is ionized, the characteristic mass and circular velocity below which accretion is suppressed should be in the range of $\sim 30-50 \mathrm{~km} / \mathrm{s}$, as was shown by many studies (Thoul \& Weinberg 1996; Ouinn et al. 1996; Navarro \& Steinmetz 1997; Kitavama \& Ikeuchi 2000; Gnedin 2000; Dijkstra et al. 2004). Therefore, we define a reionization fossil as a halo that never increased its maximum circular velocity above a given fixed threshold $V_{\text {rf }}$ (note that we use $V_{\mathrm{rf}}$ here to distinguish the threshold circular velocity for fossils from the filtering velocity, $V_{\mathrm{F}}$ ),

$$
\max \left[V_{\max }(z)\right]<V_{\text {rf }} \text {. }
$$

This definition can be easily implemented for the dissipationless simulations we use, as we track the evolution of $V_{\max }$ for all halos.

Figure 5 shows the dependence of the survival probability on this parameter for halos with $V_{\max }=20 \pm 2 \mathrm{~km} / \mathrm{s}$. In the limit of large $V_{\mathrm{rf}}$, for example, $14 \%$ of all halos present at $z=8$ survive as separate object by $z=0$ for the $d<1 \mathrm{Mpc}$ sample, while the rest grow into larger objects or get disrupted before $z=0$. For objects closer to the parent halo the survival probability decreases, since merging and disruption are expected to be more efficient in the higher density environment.

The survival probability levels off for $V_{\mathrm{rf}}>50 \mathrm{~km} / \mathrm{s}$, because if the value of the threshold $V_{\mathrm{rf}}$ is set too high above the value of $V_{\max }$ for the halos (20 km/s for Fig. 5), it becomes irrelevant. Note also that the survival probability is a weak function of the filtering velocity for $V_{\mathrm{rf}} \gtrsim 30 \mathrm{~km} / \mathrm{s}$. Our results thus are not particularly sensitive to the specific choice of $V_{\mathrm{rf}}$ 


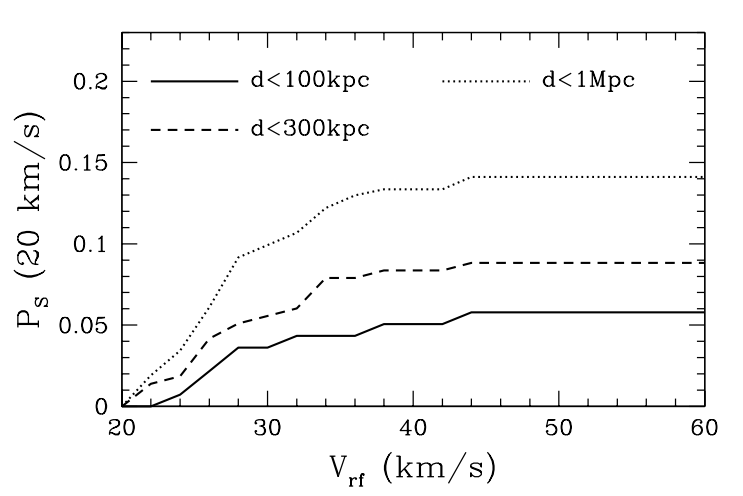

FIG. 5.- The survival probability $P_{\mathrm{S}}$ as a function of the threshold parameter $V_{\text {rf }}$ (gray line) for halos with $V_{\max }=20 \pm 2 \mathrm{~km} / \mathrm{s}$ from the L20 run. Three lines correspond to the three halo subsamples: halos within $1 \mathrm{Mpc}$ (dotted line), $300 \mathrm{kpc}$ (dashed line), and $100 \mathrm{kpc}$ of the parent halo (solid line), respectively.

as long as it is in this range. We demonstrate this explicitly below (see Fig. 77). In what follows, we adopt $V_{\mathrm{rf}}=30 \mathrm{~km} / \mathrm{s}$ as our fiducial value, as it is sufficiently small to ensure that halos that we identify as fossils have minimal accretion of fresh gas after reionization.

Figure 6 shows the survival probability as a function of $V_{\max }(z=8)$ (similar to Fig. 4) but for the reionization fossils defined using equation (2) with $V_{\mathrm{rf}}=30 \mathrm{~km} / \mathrm{s}$. Note that $P_{\mathrm{S}}$ approaches zero for $V_{\max }$ approaching the value of the threshold $V_{\text {rf }}=30 \mathrm{~km} / \mathrm{s}$, simply because the definition does not allow existence of such fossils. At $V_{\max } \lesssim 15 \mathrm{~km} / \mathrm{s}$ the incompleteness of the $d<100 \mathrm{kpc}$ sample becomes non-negligible, and the survival probability at lower values of the maximum circular velocity becomes uncertain by up to a factor of two. This uncertainty does not significantly affect our results since most of our galaxy sample has $V_{\max } \gtrsim 15 \mathrm{~km} / \mathrm{s}$.

The survival probability ranges from a few per cent for objects located close to the Milky Way host to $\approx 15 \%$ for more distant halos. Thus only a small fraction of objects at $z=8$ would survive to be true fossils at the present epoch. The survival probability distribution shown in Figure 6 is the main ingredient of our model for the luminosity function and spatial distribution of fossil galaxies presented in the next section.

\section{RESULTS}

Given the survival probability of fossils as a function of $V_{\max }$ and $d$ from the L20 run, we can use the galaxy formation run L1 to compute the luminosity function of fossils as a function of distance from the parent galaxy. Specifically, we use the distribution of high-redshift dwarfs in the $L_{V}-V_{\max }$ plane shown in Fig. 3 to compute the cumulative luminosity function of fossils at $z=0$ within a given Lagrangian region with mass $M_{\mathrm{LR}}$ :

$$
\begin{gathered}
N_{\mathrm{LR}}\left(>L_{V}\right)=\left(\frac{M_{\mathrm{LR}}}{1.2 \times 10^{11} M_{\odot}}\right) \int_{L_{V}}^{10^{7} L_{\odot}} d L \\
\int d V_{\max } P_{\mathrm{S}}\left(V_{\max }\right) \frac{d^{2} N_{8}\left(L, V_{\max }\right)}{d L d V_{\max }},
\end{gathered}
$$

where $1.2 \times 10^{11} M_{\odot}$ is the mass of the computational volume of the $\mathrm{L} 1$ run and $d^{2} N_{8}\left(L, V_{\max }\right) / d L / d V_{\max }$ is the luminosity function of high redshift dwarfs at $z=8$ in the L1 run per unit

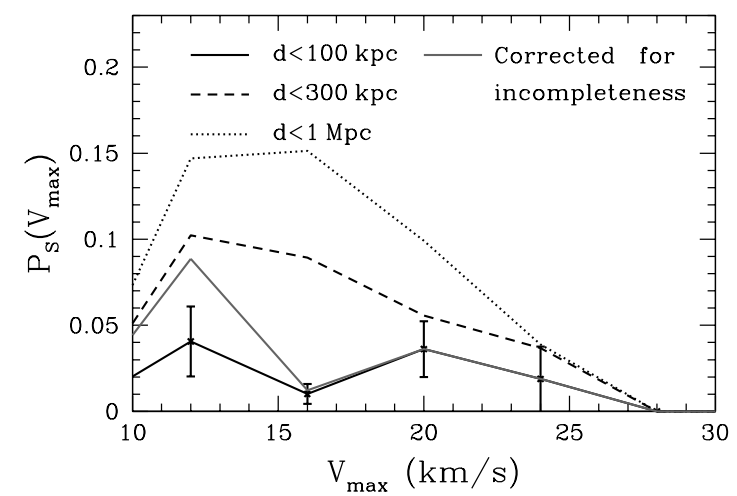

FIG. 6.- The same as in Fig. 4 but for the reionization fossils according to the definition 22. The solid gray line shows the survival probability for the $d<100 \mathrm{kpc}$ when the correction for incompleteness is taken into account.

$V_{\max }$. For the small number of objects we have in the L1 run,

$$
\frac{d^{2} N_{8}\left(L, V_{\max }\right)}{d L d V_{\max }}=\sum_{j} \delta\left(V_{\max }-V_{\max }^{j}\right) \delta\left(L_{V}-L_{V}^{j}\right),
$$

where $V_{\max }^{j}$ and $L_{V}^{j}$ are the maximum circular velocity and the V-band luminosity of a dwarf galaxy $j$.

The upper limit of $10^{7} L_{\odot}$ in the integral over the luminosity is motivated by Ricotti \& Gnedin (2005), who found that no fossils exist at higher luminosities in the Local Group. The limits of the integral over $V_{\max }$ are formally over all existing halos, but the survival probability is non-zero only over a finite range of $13 \mathrm{~km} / \mathrm{s}<V_{\max }<V_{\text {rf }}$ due to our definition of the reionization fossil and our completeness limit.

In order to define the amplitude of the cumulative luminosity function for the Local Group, we need to know the mass of the Lagrangian region that ends up within a given distance from the parent galaxy (the Milky Way or Andromeda). To estimate this mass, we use the dynamical models of the Milky Way and Andromeda from Klypin et al. (2002). For their preferred models $\left(\mathrm{A}_{1}\right.$ or $\mathrm{B}_{1}$ for the Milky Way and $\mathrm{C}_{1}$ for Andromeda), we find the total mass with $100 \mathrm{kpc}$ from the Milky Way or Andromeda (summed over these two galaxies) to be $1.4 \times 10^{12} M_{\odot}$, and the total mass within $300 \mathrm{kpc}$ to be $2.7 \times 10^{12} M_{\odot}$. For larger distances, extrapolation is required. In the L20 run, about 50\% more mass is between $300 \mathrm{kpc}$ and $1 \mathrm{Mpc}$ around the parent halo compared to the total mass within $300 \mathrm{kpc}$. This number is also consistent with the simple extrapolation of NFW profiles around the Milky Way and Andromeda. Thus, if the two galaxies were isolated, the total mass of the Local Group within $1 \mathrm{Mpc}$ would be $\approx 4.0 \times 10^{12} M_{\odot}$. However, since the distance between the Milky Way and Andromeda is about $780 \mathrm{kpc}$, there is a substantial overlap between the two spheres of $1 \mathrm{Mpc}$ in radius centered on the two galaxies, and most of the mass of both galaxies is within the overlap region. Although an accurate estimate is difficult, we adopt the mass $3.0 \times 10^{12} \mathrm{M}_{\odot}$ as a reasonable estimate of the total mass of the two galaxies within $1 \mathrm{Mpc}$ from each of them.

We first check the sensitivity of our results to adopted values of free parameters: the starting redshift $z_{s}$ at which we identify the future fossils and the fossil threshold $V_{\mathrm{rf}}$. Indeed, although $z=8$ is a reasonable choice for characteristic prereionization epoch, there is nothing special about it. This specific value is simply the epoch when the L1 run was stopped in 


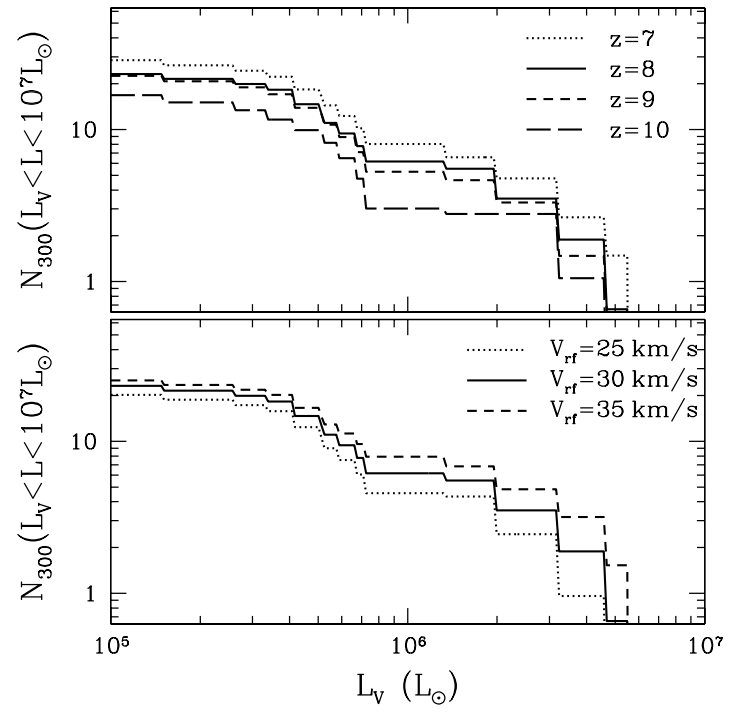

FIG. 7.- The cumulative luminosity functions of fossil dwarfs located within $300 \mathrm{kpc}$ from the parent halo at $z=0$ as a function of the starting redshift (top panel) and the fossil threshold parameter $V_{\mathrm{rf}}$ (bottom panel).

Ricotti \& Gnedin (2005) for physical and computational reasons. In addition, depending on the particular evolutionary histories of the real Milky Way and Andromeda, the Local Group region could have been reionized at different redshifts.

The top panel of Figure 7 shows the cumulative luminosity functions for fossils located within $300 \mathrm{kpc}$ of the parent halo at $z=0$, calculated using survival probabilities derived from the L20 run. Four lines correspond to the four values of starting redshift: $z_{s}=7,8,9$, and 10. Namely, the solid line in Fig. 7 is obtained using the equations above, while the dotted line $(z=7)$ is obtained from the same equations but with $z=8$ replaced by $z=7$ everywhere. The figure shows that the dependence on the specific value for the starting redshift for measuring the survival probabilities is quite weak for $z_{s} \lesssim 9$. In making this figure, we used the same $L_{V}-V_{\max }$ distribution from Fig. 3 since we cannot rerun the L1 simulation for different reionization redshifts (due to its expense). However, a preliminary analysis of different galaxy formation simulation (Tassis et al. 2006, in preparation) indicates that redshift dependence of the $L-V_{\max }$ relation for high- $z$ dwarfs is given by $L \propto V_{\max }^{3}(1+z)^{-1.5}$. Depending on how different the redshift of reionization is from our fiducial value of $z=8$, the difference in the relation can be approximated by this redshift scaling. Thus, for example, if we change redshift from $z=8$ to $z=10$ the difference in luminosity for a given $V_{\max }$ is only $35 \%$. If however, the change is to $z=15$ the difference is a factor of two. This uncertainty can therefore be assumed as uncertainty of a factor of two in the luminosity we assign to the fossil dwarfs.

At higher redshifts the dependence is much stronger. We can of course expect that as we increase the starting redshift to $z_{s} \sim 10-20$ the number of halos that do not change appreciably to the present epoch becomes very small. Our choice for the lower value of $z_{s}$ is fairly straightforward. The universe is known to be fully reionized at $z \approx 6$, so $z=7$ is quite close to the lowest possible value. Indeed, it is highly unlikely that the vicinity of the Local Group should be one of the last patches of neutral gas in the universe to be re-ionized at $z<7$. The upper limit of $z \sim 9$ is less constrained, but is generally

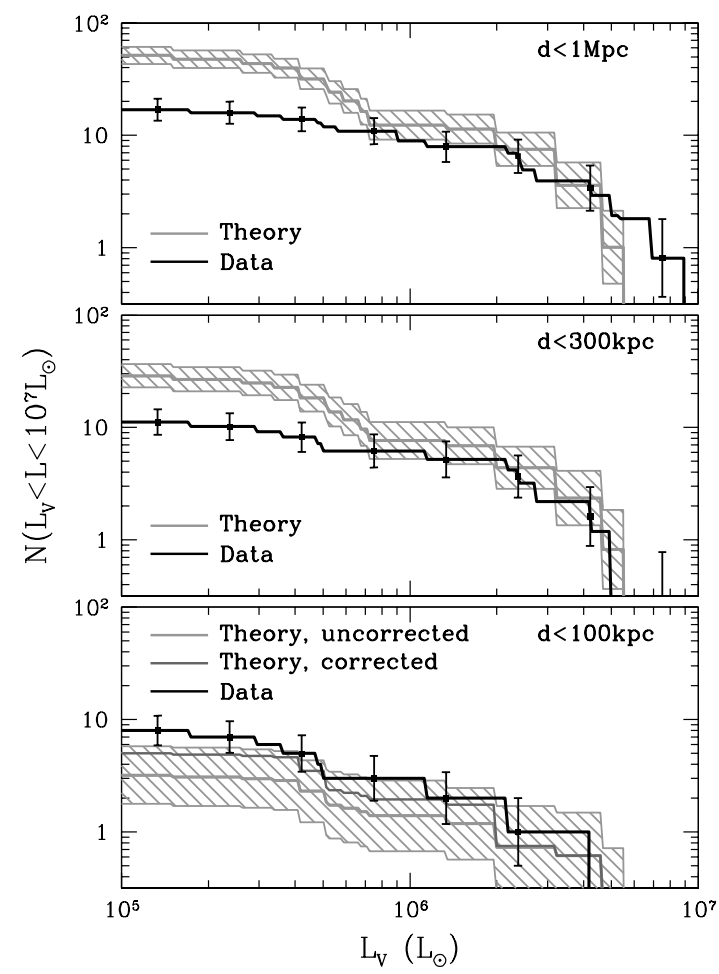

FIG. 8.- The cumulative luminosity functions of fossil dwarfs located within $1 \mathrm{Mpc}$ (top panel), $300 \mathrm{kpc}$ (middle panel), and $100 \mathrm{kpc}$ (bottom panel) from the parent galaxy at $z=0$. The black lines with error-bars are the observational data for the Local Group dwarfs, while the light gray bands show model predictions with $1 \sigma$ error-bars, uncorrected for incompleteness. The dark gray line in the bottom panel shows the model prediction corrected for the incompleteness.

a reasonable number to expect a region of about $1 h^{-1} \mathrm{Mpc}$ across to be reionized by the UV radiation from a Milky Way progenitor. Of course, other sources of radiation may cause even earlier partial or complete ionization of the Lagrangian region around the Milky Way or Andromeda. The predicted abundance of fossils would be correspondingly smaller, as we discuss in the next section. The bottom panel of Figure 7 shows dependence of the luminosity function of fossils on the adopted value of $V_{\text {rf }}$. The dependence is weak and the amplitude of the luminosity function changes by less than a factor of two for $V_{\text {rf }}$ from $25 \mathrm{~km} / \mathrm{s}$ to $35 \mathrm{~km} / \mathrm{s}$.

Figure 8 presents the main result of this paper: the cumulative luminosity function of reionization fossils in the Local Group within three different distances from the parent galaxy. The observational data for the Local Group dwarfs are taken from Mateo (1998) and McConnachie \& Irwin (2005), as described in $\S 2.2$. Since observational uncertainties on the distances (from the Milky Way) to some of Andromeda satellites are comparable to their distance to Andromeda, there is an uncertainty on whether a given dwarf galaxy is within, say, $100 \mathrm{kpc}$ from Andromeda. To take this uncertainty into account, we construct 100 random realizations of the Local Group in which dwarf spheroidal galaxies are distributed over the distance (from the Milky Way) with a log-normal distribution of the width corresponding to the distance measurement error.

The errorbars that we assign to the observed luminosity function include both the Poisson fluctuations and the error 


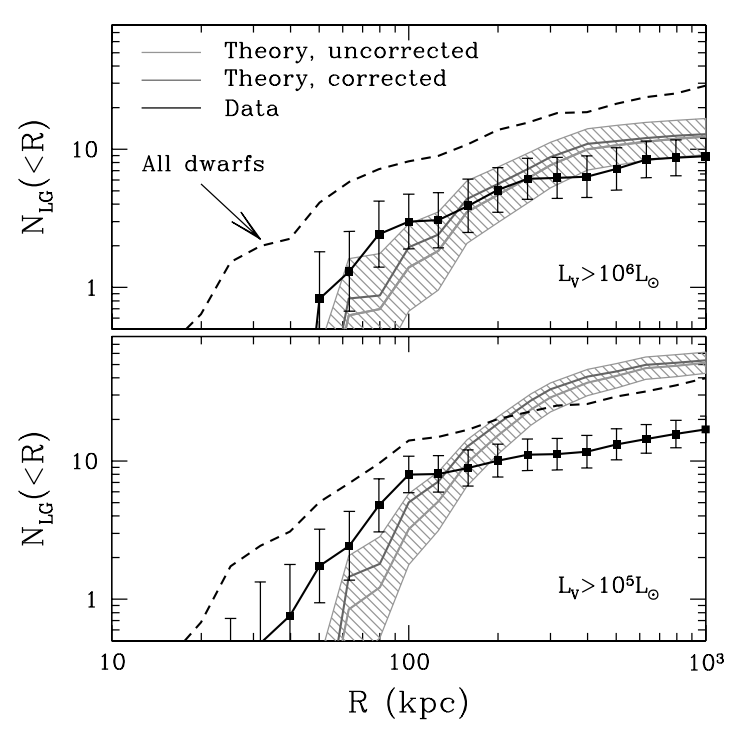

FIG. 9.- The radial distribution of reionization fossils with $L_{V}>10^{6} L_{\odot}$ (top panel) and $L_{V}>10^{5} L_{\odot}$ (bottom panel). The light gray bands and dark gray lines show theoretical expectations without and with correction for incompleteness. Black lines with error-bars show the observational data on Local Group dwarfs identified as reionization fossils by Ricotti \& Gnedin (2005), while the black dashed line show all (whether a fossil or not) Local Group dwarfs.

measured from the dispersion between 100 random realizations of galaxy distances. The predictions of our model for the fossil galaxies is shown by the hatched band and includes Poisson errors in the number of galaxies from the L1 run and Poisson errors in the survival probability due to the limited number of halos in the L20 run. Since we have only one dissipationless simulation, additional uncertainty due to cosmic variance should exist as well. However, we expect it to be smaller or comparable to the Poisson errorbars shown here. The dark gray line in the bottom panel of Fig. 8 shows the mean value of the theoretical prediction with the incompleteness correction included.

We can draw two straightforward conclusions from Figure 8

1. The expected number of fossils with $L_{V}>10^{6} L_{\odot}$ is in reasonable agreement with the observed luminosity function of the Local Group dwarfs, which are likely to be fossils, for all distances from the parent galaxy.

2. The predicted number of fossils with $10^{5} L_{\odot}<L_{V}<$ $10^{6} L_{\odot}$ is also in reasonable agreement with observations within $100 \mathrm{kpc}$, but is larger by a factor 2 to 4 for $d<300 \mathrm{kpc}$ and $d<1 \mathrm{Mpc}$.

These conclusions are not sensitive to our incompleteness correction, as it is smaller than our estimated error bars.

Figure 9 compares the predicted radial distribution of the fossil galaxies for two luminosity ranges $\left(L_{V}>10^{6} L_{\odot}\right.$ and $\left.L_{V}>10^{5} L_{\odot}\right)$ to the distribution of observed Local Group dwarfs. It is interesting to note that the distribution of observed galaxies identified by Ricotti \& Gnedin (2005) as fossils is quite similar to the radial distribution of all dwarf galaxies. There is thus no particular spatial bias exhibited by such galaxies. It is not clear whether a strong spatial bias should exist. On the one hand, the early forming high-redshift galaxies should be spatially biased with respect to their host. On the other hand, the galaxies located close to the host would be accreted early and thus may have a lower chance to survive until the present.

The figure shows that the predicted radial distribution for the brighter fossils $\left(L_{V}>10^{6} L_{\odot}\right)$ agrees with observations. For lower luminosities, however, the predicted and observed radial distributions are quite different. Although the number of predicted fossils at $d<100 \mathrm{kpc}$ is similar to the observed abundance, the observed dwarfs show a somewhat more radially concentrated distribution. The difference is not large however and is not statistically significant. At larger distances, on the other hand, the model predicts far more (a factor of 3-4) fossils with $L_{V}>10^{5} L_{\odot}$ than is observed. There is thus a marked deficit of the observed galaxies at the faintest luminosities at distances larger than $100 \mathrm{kpc}$. These conclusions are the same if we consider radial distribution for Andromeda or the Milky Way only, although the statistics is poorer in this case. We discuss possible explanations for the discrepancy in the next section.

\section{DISCUSSION AND CONCLUSIONS}

In this paper we estimate the expected abundance and spatial distribution of the reionization fossils: dwarf galaxies that formed most of their stars around $z \sim 8$ and evolved only little since then. We show that $\sim 5-15 \%$ of the objects existing at $z \sim 8$ do indeed survive until the present in the Milky Way like environment without significant evolution. Because such galaxies form their stellar systems early during the period of active merging and accretion, they should have spheroidal morphology and their observed counterparts are most likely to be identified among the dwarf spheroidal galaxies. The spheroidal morphology of the fossil galaxies is thus not related to the tidal heating by the nearby massive galaxies and they can be found even at distances as large as a megaparsec from their host. This could possibly explain existence of distant dwarf spheroidal galaxies in the Local Group, such as Cetus, Tucana, and KKR25.

We show that both the expected luminosity function and spatial distribution of dark matter halos which are likely to host fossil galaxies agrees reasonably well with the observed distribution of the luminous $\left(L_{V} \gtrsim 10^{6} L_{\odot}\right)$ Local Group fossil candidates near the host $(d \lesssim 200 \mathrm{kpc})$. The predicted abundance is substantially larger (by a factor of 2-3) for fainter galaxies $\left(L_{V}<10^{6} L_{\odot}\right)$ at larger distances $(d \gtrsim 300 \mathrm{kpc})$. This discrepancy can have at least three plausible explanations. We list them and the possible tests below.

Observations are wrong. Observational incompleteness for faint, $L_{V}<10^{6} L_{\odot}$, fossils may potentially account for the difference we find, if it can be as high as a factor of 3 at $d>300 \mathrm{kpc}$. Willman et al. (2004) found that a factor of 3 incompleteness is possible, although that number was on the very edge of their estimates. The same incompleteness factor would have to apply to the distribution of Andromeda satellites, which seems to be less likely. Future observational searches (for example, from SDSS) for dwarf spheroidals between $200 \mathrm{kpc}$ and $1 \mathrm{Mpc}$ from the Milky Way or Andromeda, or observations of nearby galaxy groups with the next generation large telescopes should be able to test this possibility.

Theory is wrong. The abundance of fossils galaxies may be overestimated by our model. This is possible for example if reionization occurs much earlier than we assumed: for example, at $z>10$, as may be suggested 
by the first year WMAP data (Kogut et al. 2003). In this case, the number of small-mass objects that are able to form stars before reionization should be much smaller. However, if most of dwarf galaxies that Ricotti \& Gnedin (2005) identified as reionization fossils are confirmed to be such by future observations, then this work will places a constraint of $z \lesssim 10$ on the reionization history of the region of the universe that collapses into the Local Group.

Spatial bias. If we take the observational data as complete and at face value, they indicate that lowest luminosity fossils $\left(L_{V} \lesssim 10^{6} L_{\odot}\right)$ are located preferentially near the parent galaxy. At first glance, such a conclusion seems counterintuitive, since the feedback (both radiative and kinetic) from the progenitor of the main galaxy is expected to suppress star formation in nearby galaxies. However, galaxies are also biased, more so at high redshift, so dwarf galaxies located closer to the progenitor of a large spiral galaxy will start forming sooner than similar galaxies in more distant regions of space. Which of the two opposing effects (feedback vs bias) wins is unclear, but should be tackled with the future ultra high-resolution cosmological simulations.

The results of this study indicate that existence of the fossil galaxies in the Local Group, in which most of the stars formed during the earliest stages of galaxy formation, is plausible.
The abundance of fossils predicted by our model would constitute as much as $20-40 \%$ of all the luminous Local Group dwarfs. The improvement in resolution of cosmological simulations should improve our estimates. Observationally, identification and studies of the fossil galaxies can provide an exciting opportunity of studying the properties of star formation in primeval galaxies by direct observations in our own cosmological backyard.

We would like to thank Anatoly Klypin for providing us the dark matter simulation of the Milky Way-sized halo used in this study. We are grateful to Oleg Gnedin, James Bullock, Brant Robertson, and Ben Moore for the comments on the draft of this paper. This work was supported in part by the DOE and the NASA grant NAG 5-10842 at Fermilab, by the HST Theory grant HST-AR-10283.01, by the NSF grants AST-0206216, AST-0239759, and AST-0507596, and by the Kavli Institute for Cosmological Physics at the University of Chicago. Supercomputer simulations were run on the IBM P690 array at the National Center for Supercomputing Applications (under grant AST-020018N) and the Sanssouci computing cluster at the Astrophysikalisches Institut Potsdam. We are grateful to the King Kamehameha Beach Hotel in Kona for hospitality during the initial stages of this project. This work made extensive use of the NASA Astrophysics Data System and arXiv. org preprint server.

\section{REFERENCES}

Barkana, R. \& Loeb, A. 1999, ApJ, 523, 54

Benson, A. J., Frenk, C. S., Lacey, C. G., Baugh, C. M., \& Cole, S. 2002, MNRAS, 333, 177

Bullock, J. S. \& Johnston, K. V. 2005, ApJ, 635, 931

Bullock, J. S., Kravtsov, A. V., \& Weinberg, D. H. 2000, ApJ, 539, 517

-. 2001, ApJ, 548, 33

Dijkstra, M., Haiman, Z., Rees, M. J., \& Weinberg, D. H. 2004, ApJ, 601, 666

Efstathiou, G. 1992, MNRAS, 256, 43P

Gnedin, N. Y. 2000, ApJ, 542, 535

Gnedin, N. Y. \& Hui, L. 1998, MNRAS, 296, 44

Kauffmann, G., White, S. D. M., \& Guiderdoni, B. 1993, MNRAS, 264, 201

Kazantzidis, S., Mayer, L., Mastropietro, C., Diemand, J., Stadel, J., \& Moore, B. 2004, ApJ, 608, 663

Kitayama, T. \& Ikeuchi, S. 2000, ApJ, 529, 615

Klypin, A., Kravtsov, A. V., Valenzuela, O., \& Prada, F. 1999, ApJ, 522, 82

Klypin, A., Zhao, H., \& Somerville, R. S. 2002, ApJ, 573, 597

Kogut, A., Spergel, D. N., Barnes, C., Bennett, C. L., Halpern, M., Hinshaw, G., Jarosik, N., Limon, M., Meyer, S. S., Page, L., Tucker, G. S., Wollack, E., \& Wright, E. L. 2003, ApJS, 148, 161

Kravtsov, A. V. 1999, PhD thesis, New Mexico State University

Kravtsov, A. V. \& Gnedin, O. Y. 2005, ApJ, 623, 650

Kravtsov, A. V., Gnedin, O. Y., \& Klypin, A. A. 2004, ApJ, 609, 482

Kravtsov, A. V., Klypin, A. A., \& Khokhlov, A. M. 1997, ApJS, 111, 73

Macciò, A. V., Moore, B., Stadel, J., \& Diemand, J. 2005, ArXiv Astrophysics e-prints

Mateo, M. L. 1998, ARA\&A, 36, 435

McConnachie, A. W. \& Irwin, M. J. 2005, astro-ph, 0510654

Moore, B., Diemand, J., Madau, P., Zemp, M., \& Stadel, J. 2005, MNRAS submitted (astro-ph/0510370)

Moore, B., Ghigna, S., Governato, F., Lake, G., Quinn, T., Stadel, J., \& Tozzi, P. 1999, ApJ, 524, L19
Navarro, J. F. \& Steinmetz, M. 1997, ApJ, 478, 13

Prada, F., Klypin, A. A., Simonneau, E., Betancort-Rijo, J., Patiri, S., Gottloeber, S., \& Sanchez-Conde, M. A. 2005, ApJ submitted (astro$\mathrm{ph} / 0506432$ )

Quinn, T., Katz, N., \& Efstathiou, G. 1996, MNRAS, 278, L49

Reed, D., Governato, F., Quinn, T., Gardner, J., Stadel, J., \& Lake, G. 2005, MNRAS, 359, 1537

Ricotti, M. \& Gnedin, N. Y. 2005, ApJ, 629, 259

Ricotti, M., Gnedin, N. Y., \& Shull, J. M. 2002a, ApJ, 575, 33

-. 2002b, ApJ, 575, 49

Robertson, B., Bullock, J. S., Font, A. S., Johnston, K. V., \& Hernquist, L. 2005, ApJ, 632, 872

Shapiro, P. R., Iliev, I. T., \& Raga, A. C. 2004, MNRAS, 348, 753

Shaviv, N. J. \& Dekel, A. 2003, MNRAS submitted, astro-ph/0305527

Somerville, R. S. 2002, ApJ, 572, L23

Stoehr, F., White, S. D. M., Tormen, G., \& Springel, V. 2002, MNRAS, 335, L84

Tasitsiomi, A. 2006, ApJ, in press, TBD

Taylor, J. E., Silk, J., \& Babul, A. 2004, in IAU Symposium (astro$\mathrm{ph} / 0312086), 91$

Thoul, A. A. \& Weinberg, D. H. 1996, ApJ, 465, 608

White, S. D. M. \& Springel, V. 2000, in The First Stars. Proceedings of the MPA/ESO Workshop held at Garching, Germany, 4-6 August 1999. Achim Weiss, Tom G. Abel, Vanessa Hill (eds.). Springer, p.327, 327

Willman, B., Governato, F., Dalcanton, J. J., Reed, D., \& Quinn, T. 2004, MNRAS, 353, 639

Zentner, A. R. \& Bullock, J. S. 2003, ApJ, 598, 49 